\title{
The radical S-adenosyl---methionine enzyme MftC catalyzes an oxidative decarboxylation of the C-terminus of the MftA peptide
}

\author{
Nathan A. Bruender and Vahe Bandarian* \\ Department of Chemistry, University of Utah, Salt Lake City, Utah 84112, United States
}

\begin{abstract}
Ribosomally-synthesized post-translationally modified peptides, RiPPs, are encoded in the genomes of a wide variety of microorganisms, in close proximity to orfs that encode enzymes that carry out extensive modifications, many of which are novel. Recently, members of the radical $S$ adenosyl-L-methionine (SAM) superfamily have been identified in these biosynthetic clusters. Herein we demonstrate the putative radical SAM enzyme, MftC, oxidatively decarboxylates the Cterminus of the MftA peptide in the presence of the accessory protein MftB. The reaction catalyzed by MftC expands the repertoire of peptide-based radical SAM chemistry beyond the intramolecular crosslinks.
\end{abstract}

\section{Graphical Abstract}<smiles>CC(N)C(NC(C)(F)F)C(=O)O</smiles>

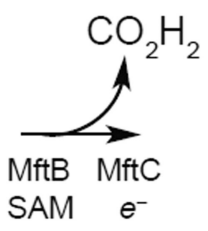<smiles>CN/C=C\c1ccc(O)cc1</smiles>

Ribosomally-synthesized post-translationally modified peptides (RiPPs) are a class of peptide derived natural products that are increasingly being identified in microorgansims. ${ }^{1}$ The mature RiPPs are derived from a precursor peptide, which is encoded by an orf in the genome, that is often extensively modified post-translationally. ${ }^{1}$ Interestingly, the orfs encoding for the modification enzymes are often clustered near the orf encoding for the precursor peptide in the genome of many RiPP producing organisms. ${ }^{1}$

\footnotetext{
*Corresponding Author. vahe@ chem.utah.edu. Phone: (801) 581-6366. Fax: (801) 581-8433. ASSOCIATED CONTENT

The Supporting Information is available free of charge on the ACS Publications website. A single PDF contains the materials and methods, Table S1-S3, and Figures S1-S7.

Author Contributions

NAB and VB designed the experiments and wrote the manuscript. NAB performed all experiments.

No competing financial interests have been declared.
} 
Recently, orfs encoding members of the radical $S$-adenosyl-L-methionine superfamily have been observed near orfs encoding for peptides suggesting that the radical SAM enzymes post-translationally modify the peptides. ${ }^{1-5}$ Majority of the members of the superfamily are primarily identified on the basis of a $\mathrm{CxxxCxxC}$ motif, which coordinate three iron atoms of a [ $4 \mathrm{Fe}-4 \mathrm{~S}]$ cluster. ${ }^{6}$ The unique fourth iron is coordinated by the $\mathrm{a}$-amino and $\mathrm{a}$-carboxylate moieties of SAM. ${ }^{7,8}$ Upon reduction to the +1 oxidation state from the resting +2 oxidation state, the cluster mediates the reductive cleavage of SAM; the resulting SAM-derived radical species is used to abstract a hydrogen atom to initiate catalysis. ${ }^{9}$ To date, members of the radical SAM superfamily have been shown to install Lys to Trp crosslinks, ${ }^{10} \mathrm{Glu}$ to Tyr crosslinks, ${ }^{11}$ and thioether crosslinks between a Cys sidechain and the $\mathrm{C}_{a}$ or $\mathrm{C}_{\beta}$ of peptides, ${ }^{5,12-16}$ in the biosynthesis of several RiPPs.

We were intrigued by a bioinformatic investigation that identified instances where a radical SAM enzyme co-localizes with an orf that encodes a peptide and several additional proteins of unknown function. ${ }^{2}$ The nearby orfs encode for putative maturases that have sequence similarity to a radical SAM enzyme, heme/flavin dehydrogenase, creatininase, and glycosyltransferase, respectively. (Scheme 1).

Bioinformatic analysis shows that three of the orfs ( $m f t A, m f t B$, and $m f t C$ ) are clustered in at least 336 genomes (based on the interpro family IPR023850 for the $m f t B$ gene product $\mathrm{MftB}) .{ }^{17}$ Interestingly, the clustering of these three orfs is reminiscent of the orf clustering in the biosynthesis of the redox cofactor, pyrroloquinoline quinone (PQQ).${ }^{18-20}$ In that pathway, the $p q q D$ and $p q q E$ genes encode proteins that initiate the conversion of the precursor peptide PqqA to PQQ. ${ }^{11,21}$ The $p q q E$ gene encodes a radical SAM enzyme that installs a crosslink between Glu15 and Tyr19 in PqqA in a PqqD-dependent manner in the first step of the PQQ biosynthetic pathway. ${ }^{11}$

The $m f t$ cluster of genes often occurs near dehydrogenases, prompting the suggestion that this cluster carries out transformations on the peptide, MftA, encoded by the co-localized orf, to generate a putative redox cofactor used by the downstream dehydrogenase enzymes. ${ }^{2}$ Since this orf is most often found in mycobacterial species, the as yet unidentified factor was termed mycofactocin and the putative maturases for the peptide, MftA, were termed MftB and $\mathrm{MftC} .^{2}$

To determine the biological function of the $\mathrm{mft}$ gene cluster we cloned $\mathrm{mft} C$ from Mycobacterium smegmatis and recombinantly expressed the corresponding protein. The $m f t C$ gene encodes for a member of the radical SAM superfamily based on the presence of the aforementioned $\mathrm{CxxxCxxC}$ motif. ${ }^{6}$ The as-isolated protein, which was anaerobically purified using a $\mathrm{N}$-terminal $\mathrm{His}_{6}$-tag, is brown in color. The UV-visible spectrum contains a feature at $420 \mathrm{~nm}$, which is consistent with the presence of iron-sulfur clusters (Fig S1A, black). Upon reconstitution with excess iron and sulfide the feature at $420 \mathrm{~nm}$ in the UVvisible spectrum increased indicating an increased presence of iron-sulfur clusters in the protein (Fig S1A, red). Recombinant MftC was judged to be $₫ 95 \%$ pure determined by SDS-PAGE (Fig S1B). Iron and sulfide analyses show that purified and reconstituted protein contains $10.8 \pm 1.6 \mathrm{~mol}$ of iron and $7.4 \pm 2.0 \mathrm{~mol}$ of sulfide per mol of $\mathrm{MftC}$. 
In the NCBI database, the MftA peptide is observed at varying lengths in mycobacterial homologs ranging from $\sim 30$ to $>50$ amino acids and in M. smegmatis there are two MftA precursor peptides annotated that have lengths of 31 and 52 amino acids. Interestingly, the last 31 amino acids of the longer peptide are identical to the anno-tated shorter peptide. Therefore, we synthesized the shorter putative peptide substrate, MftA, by solid phase peptide synthesis using Fmoc chemistry and we omitted the N-terminal Met residue of the annotated MftA peptide (GI:777215564) (Fig S2, see Scheme 1 for the sequence).

We first examined whether MftC catalyzes the reduc-tive cleavage of SAM. In these experiments, MftC was combined with SAM in the presence of MftA and dithionite and the formation of dAdo was examined by LC-MS. As shown in Fig. 1, in the presence of MftC we observe a peak with the expected mass for dAdo.

Next we examined if MftC modifies MftA under reducing conditions in the presence or absence of SAM. Interestingly, while MftC clearly is able to reductively cleave SAM under these conditions (Fig. 1), we observe no modification of the MftA peptide. Fig. 2A shows the region of the MftA mass spectrum corresponding to the +2 charge state in the control reaction lacking SAM (see Fig. S6A for the full spectrum). In the deconvoluted mass spectrum we observe a peak at 3320.5149 amu corresponding to the MftA peptide (theoretical 3320.5185, $1.1 \mathrm{ppm}$ error) (Fig 2B). As is shown in Fig. 2C and 2D, the inclusion of SAM does not change the spectrum of MftA. Additionally, the only change observed in the HPLC chromatogram, monitored at $280 \mathrm{~nm}$, is the presence of dAdo upon incubating MftA with MftC in the presence of SAM and dithionite (see Fig. S4A). This observation is interesting because in the PQQ system, it has been recognized that the radical SAM enzyme PqqE alone is not enough for initiating the biosynthetic pathway, and that an accessory protein, $\mathrm{PqqD}$, is also essential. ${ }^{11}$ While there is no protein in the $\mathrm{mft}$ cluster that shares significant sequence similarity with PqqD by standard BLAST alignments, Burkhart et al., demonstrated that the MftB sequence was similar to PqqD using HHpred suggesting it contains a RiPP recognition element. ${ }^{22}$

To assess if MftB is also required for MftC to post-translationally modify MftA, it was expressed and purified ( $\geq 90 \%$ purity as determined by SDS-PAGE, Fig S1B) as described in the materials and methods (see Supporting Information). When incubated with MftA in the presence of SAM but in the absence of MftC we observe the formation of no dAdo (Fig 1) and no change in the mass spectrum of the peptide (Fig 2E, 2F, and S6A). However, when both MftB and MftC are included in the reaction mixtures, a new peak is observed in the mass spectrum with a monoisotopic mass of $1637.7555 \mathrm{amu}$ for the +2 charge state mass envelope (Fig. 2G and Table S1), which is $23.0034 \mathrm{amu}$ lighter than that of the unmodified MftA (see Fig S6A for the full mass spectrum). Since the observed mass difference is for the +2 charge state, the shift in the peptide corresponds to 46.0068 ати $(23.0034 * 2=46.0068)$. In the reaction, both the new product and the MftA substrate co-elute (see Fig S4B and S4C) but are clearly distinguished in the mass spectrum. The deconvoluted spectra of unmodified (Fig. 2H) and modified MftA (Fig. 2H) show that mass of the peptide is reduced by 46.0076 атu in the presence of MftB, MftC, and SAM. The change in the mass of MftA suggests the loss of $\mathrm{CO}_{2} \mathrm{H}_{2}$ (theoretical 46.0055). The monoisotopic masses for both the unmodified 
MftA and the modified MftA, which loses $\mathrm{CO}_{2} \mathrm{H}_{2}$, are within $3.9 \mathrm{ppm}$ error of the theoretical masses of the corresponding charge states (see Table S1).

To determine the location of the modification in the MftA product, the +3 charge state corresponding to the unmodified and modified MftA were subjected to higher energy collision dissociation (HCD) fragmentation. The $20 b$-ions in the MS/MS spectrum of modified MftA all exhibit the same $\mathrm{m} / \mathrm{z}$ as the $20 b$-ions detected in the MS/MS spectrum of the unmodified peptide (Fig S7, Table S3). The smallest observed $y$-ion from the fragmentation of the modified MftA is $y_{2}$, which shows that the loss of $46.0058 \mathrm{amu}$ originates from one of the two C-terminal residues ( $\mathrm{Val}_{29}$ and/or $\left.\mathrm{Tyr}_{30}\right)$. All other $11 y$-ions observed in the MS/MS spectrum of the modified peptide show the loss of $\mathrm{CO}_{2} \mathrm{H}_{2}(\mathrm{Fig} \mathrm{S} 7 \mathrm{~B}$, Table S3).

The most likely source of the $\mathrm{CO}_{2} \mathrm{H}_{2}$ from the terminal two amino acids is the $\mathrm{C}$-terminal carboxylate group of $\mathrm{Tyr}_{30}$. Based on the mass loss and fragmentation of the modified MftA we reasoned that $\mathrm{MftC}$ catalyzes a rad-ical-mediated oxidative decarboxylation of the $\mathrm{C}$ terminal Tyr in MftA. To probe if $\mathrm{MftC}$ does indeed catalyze the oxidative decarboxylation of MftA, we synthesized a MftA peptide containing $\left[{ }^{13} \mathrm{C}_{9},{ }^{15} \mathrm{~N}_{1}\right]$-Tyr at the C-terminus only using Fmoc- $\left[{ }^{13} \mathrm{C}_{9},{ }^{15} \mathrm{~N}_{1}\right]$-Tyr. The mass of the product of the peptide $\left(\left[{ }^{13} \mathrm{C}_{9},{ }^{15} \mathrm{~N}_{1}\right]-\mathrm{Tyr}_{30^{-}}\right.$ MftA) is 10.0148 amu greater than that of unlabeled MftA (observed 3330.5493, theoretical $3330.5457,1.1 \mathrm{ppm}$ error), consistent with the added isotopes (see Fig S3). Incubation of $\left[{ }^{13} \mathrm{C}_{9},{ }^{15} \mathrm{~N}_{1}\right]-\mathrm{Tyr}_{30}-\mathrm{MftA}$ with MftB, MftC, and dithionite, was carried out in the presence or absence of SAM. When SAM is not present, there are no changes in the HPLC chromatogram, monitored at $280 \mathrm{~nm}$, or mass of the MftA present in the assay (Fig S5 and Fig S6B). However, when SAM is included we observe a shift in the mass of the peptide (Fig S6B). For clarity, we are only showing the +2 charge state in Fig. 2I, which highlights a $23.5041 \mathrm{amu}$ loss relative to the labeled peptide substrate. Fig 2J shows the deconvoluted mass spectrum generated from the full mass spectrum (Fig S6B) for the reaction containing SAM. The $m / z 3330.5303$ corresponds to the unmodified $\left[{ }^{13} \mathrm{C}_{9},{ }^{15} \mathrm{~N}_{1}\right]-\mathrm{Tyr}_{30}-\mathrm{MftA}$ (theoretical $\mathrm{m} / z 3330.5457,4.6 \mathrm{ppm}$ error). The isotopically labeled-Tyr $30-\mathrm{MftA} *$ product has a $\mathrm{m} / \mathrm{z} 3283.5220$, which is 47.0083 amu lighter than the unmodified isotopically enriched peptide. The species lost from the $\left[{ }^{13} \mathrm{C}_{9},{ }^{15} \mathrm{~N}_{1}\right]-\mathrm{Tyr}_{30}$-MftA substrate is $1.0007 \mathrm{amu}$ heavier than the species lost from the natural abundance MftA peptide. This is consistent with the $\mathrm{CO}_{2} \mathrm{H}_{2}$ being lost from the $\mathrm{C}$-terminal Tyr and represents the loss of the C-terminal carboxylate group of MftA. However, it is not known whether the resulting alkene adopts the $E$ - or Z-configuration.

These data collectively indicate that $\mathrm{MftC}$ catalyzes the oxidative decarboxylation of the $\mathrm{MftA}$ peptide in a reaction that requires the presence of MftB. The role of MftB in this system is not known. However, it may be playing a role analogous to PqqD by binding the peptide substrate to facilitate binding to the $\mathrm{MftC}$ for chemistry. ${ }^{11,22}$

Fig 3 shows a putative mechanism for the reaction catalyzed by MftC. We propose that MftC abstracts a hydrogen atom from the $\beta$-carbon of the $\mathrm{C}$-terminal Tyr residue. The resulting radical species is stabilized by the adjacent phenol ring. One can envision at least two plausible routes both ending with the oxidative decarboxylation of the C-terminus. The top 
pathway shows transfer of the unpaired spin from the radical intermediate to a [4Fe-4S] cluster concomitant with decarboxylation to form the final product. Alternatively, the $\mathrm{C}_{\mathrm{a}}-$ $\mathrm{COOH}$ bond can be homolytically cleaved resulting in the formation of a " $\bullet \mathrm{COOH}$ " species that can either be quenched to formate or $\mathrm{CO}_{2}$.

Oxidative decarboxylations are not unique to the Mft system and have been observed previously in the biosynthesis of the lanthipeptide EpiA, by the flavoenzyme EpiD, and in the radical-mediated rearrangements catalyzed by the radical SAM enzymes TYW1, HemN, and AhbD. ${ }^{23-27}$ However, this report marks the first instance of a radical SAM enzyme catalyzing an oxidative decarboxylation of a peptide and not installing intramolecular crosslinks, ${ }^{5,10-16}$ thus expanding the repertoire of peptide-based radical SAM chemistry.

We hypothesize that the reaction catalyzed by $\mathrm{MftC}$ represents a likely first step in the posttranslational modification of MftA. It is possible that MftA is further processed by one or more enzymes encoded by the genes localized near the $m f t A, m f t B$, and $m f t C$ genes. If MftA is indeed a precursor to a novel cofactor as suggested by $\mathrm{Haft}^{2}{ }^{2}$ the oxidative decarboxylated C-terminal tyrosine residue would need to be removed from the precursor peptide by a peptidase analogous to the proposed processing of PQQ to obtain the small molecule redox metabolite. Studies to probe the role of the remaining orfs in the cluster are currently underway.

\section{Supplementary Material}

Refer to Web version on PubMed Central for supplementary material.

\section{Acknowledgments}

Funding Sources

The work reported in this publication was supported by National Institutes of General Medical Sciences of the National Institutes of Health grant R01 GM72623 awarded to VB. The content is solely the responsibility of the authors and does not necessarily represent the official views for the National Institutes of Health.

\section{ABBREVIATIONS}

$\begin{array}{ll}\text { dAdo } & \text { 5'-deoxyadenosine } \\ \text { HCD } & \text { higher energy collision dissociation } \\ \text { orf } & \text { open reading frame } \\ \text { PQQ } & \text { pyrroloquinoline quinone } \\ \text { RiPP } & \text { ribosomally-synthesized posttranslationally modified peptide } \\ \text { SAM } & \text { S-adenosyl-L-methionine }\end{array}$

\section{REFERENCES}

1. Arnison PG, Bibb MJ, Bierbaum G, Bowers AA, Bugni TS, Bulaj G, Camarero JA, Campopiano DJ, Challis GL, Clardy J, Cotter PD, Craik DJ, Dawson M, Dittmann E, Donadio S, Dorrestein PC, 
Entian KD, Fischbach MA, Garavelli JS, Goransson U, Gruber CW, Haft DH, Hemscheidt TK, Hertweck C, Hill C, Horswill AR, Jaspars M, Kelly WL, Klinman JP, Kuipers OP, Link AJ, Liu W, Marahiel MA, Mitchell DA, Moll GN, Moore BS, Muller R, Nair SK, Nes IF, Norris GE, Olivera BM, Onaka H, Patchett ML, Piel J, Reaney MJ, Rebuffat S, Ross RP, Sahl HG, Schmidt EW, Selsted ME, Severinov K, Shen B, Sivonen K, Smith L, Stein T, Sussmuth RD, Tagg JR, Tang GL, Truman AW, Vederas JC, Walsh CT, Walton JD, Wenzel SC, Willey JM, van der Donk WA. Nat Prod Rep. 2013; 30:108-160. [PubMed: 23165928]

2. Haft DH. BMC genomics. 2011; 12:21. [PubMed: 21223593]

3. Haft DH, Basu MK. J Bacteriol. 2011; 193:2745-2755. [PubMed: 21478363]

4. Murphy K, O'Sullivan O, Rea MC, Cotter PD, Ross RP, Hill C. PloS one. 2011; 6:e20852. [PubMed: 21760885]

5. Bruender NA, Wilcoxen J, Britt RD, Bandarian V. Biochemistry. 2016; 55:2122-2134. [PubMed: 27007615]

6. Sofia HJ, Chen G, Hetzler BG, Reyes-Spindola JF, Miller NE. Nucleic Acids Res. 2001; 29:10971106. [PubMed: 11222759]

7. Walsby CJ, Ortillo D, Broderick WE, Broderick JB, Hoffman BM. J Am Chem Soc. 2002; 124:11270-11271. [PubMed: 12236732]

8. Chen D, Walsby C, Hoffman BM, Frey PA. J Am Chem Soc. 2003; 125:11788-11789. [PubMed: 14505379]

9. Broderick JB, Duffus BR, Duschene KS, Shepard EM. Chem Rev. 2014; 114:4229-4317. [PubMed: 24476342]

10. Schramma KR, Bushin LB, Seyedsayamdost MR. Nat Chem. 2015; 7:431-437. [PubMed: 25901822]

11. Barr I, Latham JA, Iavarone AT, Chantarojsiri T, Hwang JD, Klinman JP. J Biol Chem. 2016; 291:8877-8884. [PubMed: 26961875]

12. Ono K, Okajima T, Tani M, Kuroda S, Sun D, Davidson VL, Tanizawa K. J Biol Chem. 2006; 281:13672-13684. [PubMed: 16546999]

13. Flühe L, Knappe TA, Gattner MJ, Schafer A, Burghaus O, Linne U, Marahiel MA. Nat Chem Biol. 2012; 8:350-357. [PubMed: 22366720]

14. Flühe L, Burghaus O, Wieckowski BM, Giessen TW, Linne U, Marahiel MA. J Am Chem Soc. 2013; 135:959-962. [PubMed: 23282011]

15. Wieckowski BM, Hegemann JD, Mielcarek A, Boss L, Burghaus O, Marahiel MA. FEBS lett. 2015; 589:1802-1806. [PubMed: 26026269]

16. Nakai T, Ito H, Kobayashi K, Takahashi Y, Hori H, Tsubaki M, Tanizawa K, Okajima T. J Biol Chem. 2015; 290:11144-11166. [PubMed: 25778402]

17. Mitchell A, Chang H-Y, Daugherty L, Fraser M, Hunter S, Lopez R, McAnulla C, McMenamin C, Nuka G, Pesseat S, Sangrador-Vegas A, Scheremetjew M, Rato C, Yong S-Y, Bateman A, Punta M, Attwood TK, Sigrist CJA, Redaschi N, Rivoire C, Xenarios I, Kahn D, Guyot D, Bork P, Letunic I, Gough J, Oates M, Haft D, Huang H, Natale DA, Wu CH, Orengo C, Sillitoe I, Mi H, Thomas PD, Finn RD. Nucleic Acids Res. 2015; 43:D213-D221. [PubMed: 25428371]

18. Goosen N, Horsman HP, Huinen RG, van de Putte P. J Bacteriol. 1989; 171:447-455. [PubMed: 2536663]

19. Velterop JS, Sellink E, Meulenberg JJ, David S, Bulder I, Postma PW. J Bacteriol. 1995; 177:5088-5098. [PubMed: 7665488]

20. Toyama H, Chistoserdova L, Lidstrom ME. Microbiol. 1997; 143:595-602.

21. Bonnot F, Iavarone AT, Klinman JP. Biochemistry. 2013; 52:4667-4675. [PubMed: 23718207]

22. Burkhart BJ, Hudson GA, Dunbar KL, Mitchell DA. Nat Chem Biol. 2015; 11:564-570. [PubMed: 26167873]

23. Kupke T, Kempter C, Gnau V, Jung G, Götz F. J Biol Chem. 1994; 269:5653-5659. [PubMed: 8119901]

24. Kupke T, Kempter C, Jung G, Götz F. J Biol Chem. 1995; 270:11282-11289. [PubMed: 7744764]

25. Young AP, Bandarian V. Biochemistry. 2011; 50:10573-10575. [PubMed: 22026549] 
26. Lobo SAL, Lawrence AD, Romão CV, Warren MJ, Teixeira M, Saraiva LM. Biochim Biophys Acta. 2014; 1844:1238-1247. [PubMed: 24713144]

27. Layer G, Verfürth K, Mahlitz E, Jahn D. J Biol Chem. 2002; 277:34136-34142. [PubMed: 12114526] 


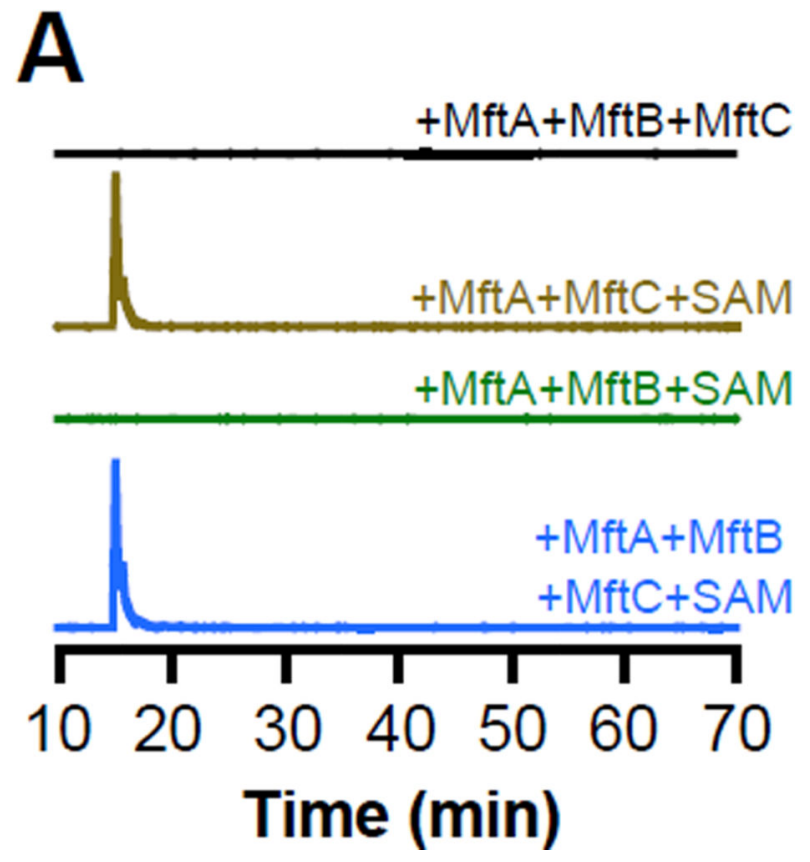

B

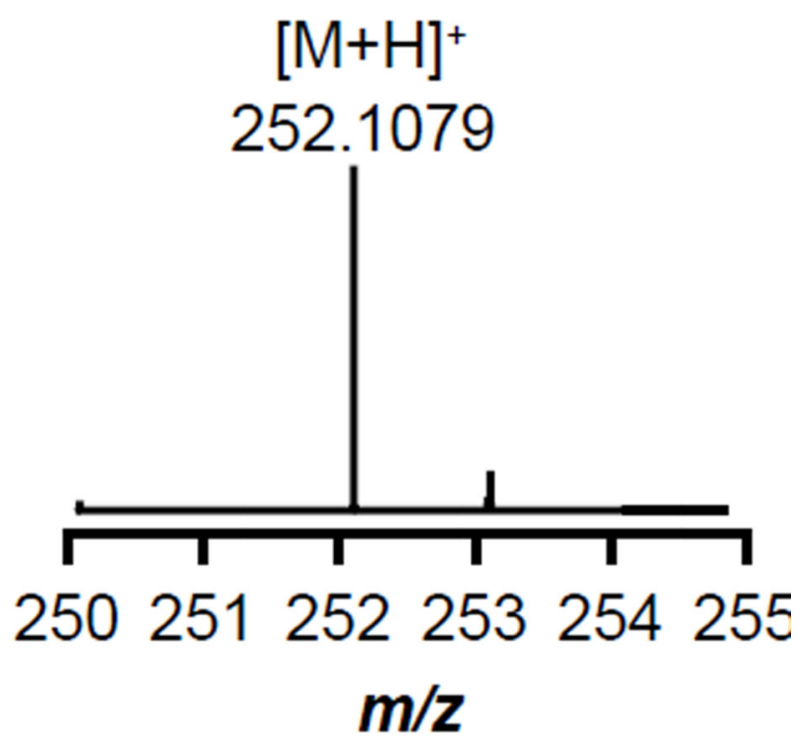

Figure 1.

(A) Extracted ion chromatogram corresponding to dAdo $(\mathrm{m} / \mathrm{z}=251.5-252.5)$. dAdo is only formed when MftC and SAM are present in the reaction. MftB has no effect on the formation of dAdo showing that $\mathrm{MftC}$ alone is capable of catalyzing the reductive cleavage of SAM. (B) Mass spectrum of dAdo eluting ca. $15 \mathrm{~min}$. 


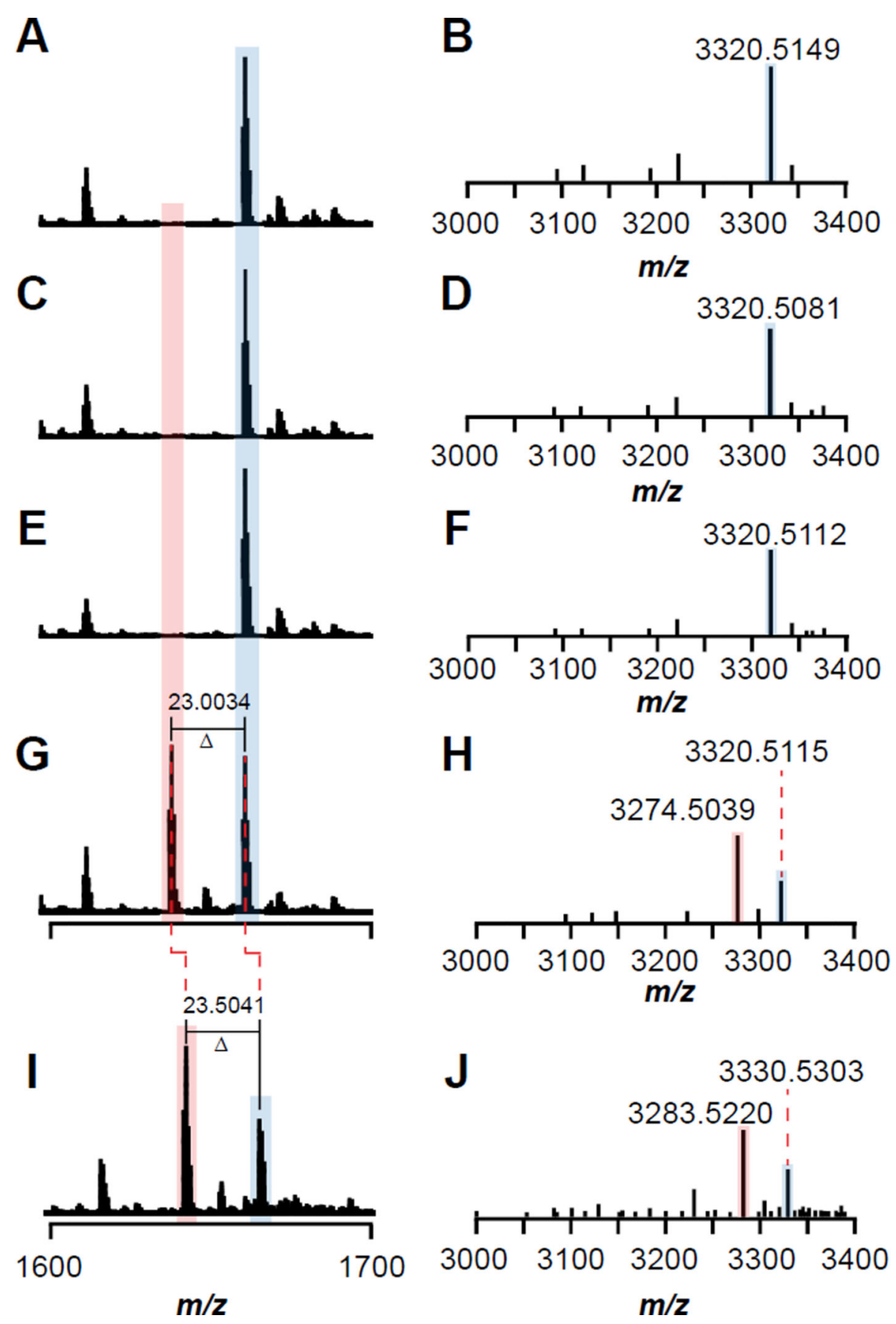

Figure 2.

The mass spectrum zoomed in on the +2 charge state mass envelope and the corresponding deconvoluted mass spectrum of unlabeled MftA isolated from reactions where either SAM (A and B), MftB (C and D), or MftC ( $\mathbf{E}$ and $\mathbf{F}$ ) were omitted. $\mathbf{G}$ and $\mathbf{H}$ correspond to the peptide isolated from the reaction where unlabeled MftA was incubated in the presence of all three components. I and $\mathbf{J}$ correspond to the peptide isolated from the reaction where $\left[{ }^{13} \mathrm{C}_{9},{ }^{15} \mathrm{~N}\right]-\mathrm{Tyr}_{30} \mathrm{MftA}$ was incubated in the presence of MftB, MftC, and SAM. The blue and red boxes highlight the peaks corresponding to unmodified and modified MftA 
respectively. The deconvoluted mass spectra were generated from the full mass spectra shown in Fig S6. 

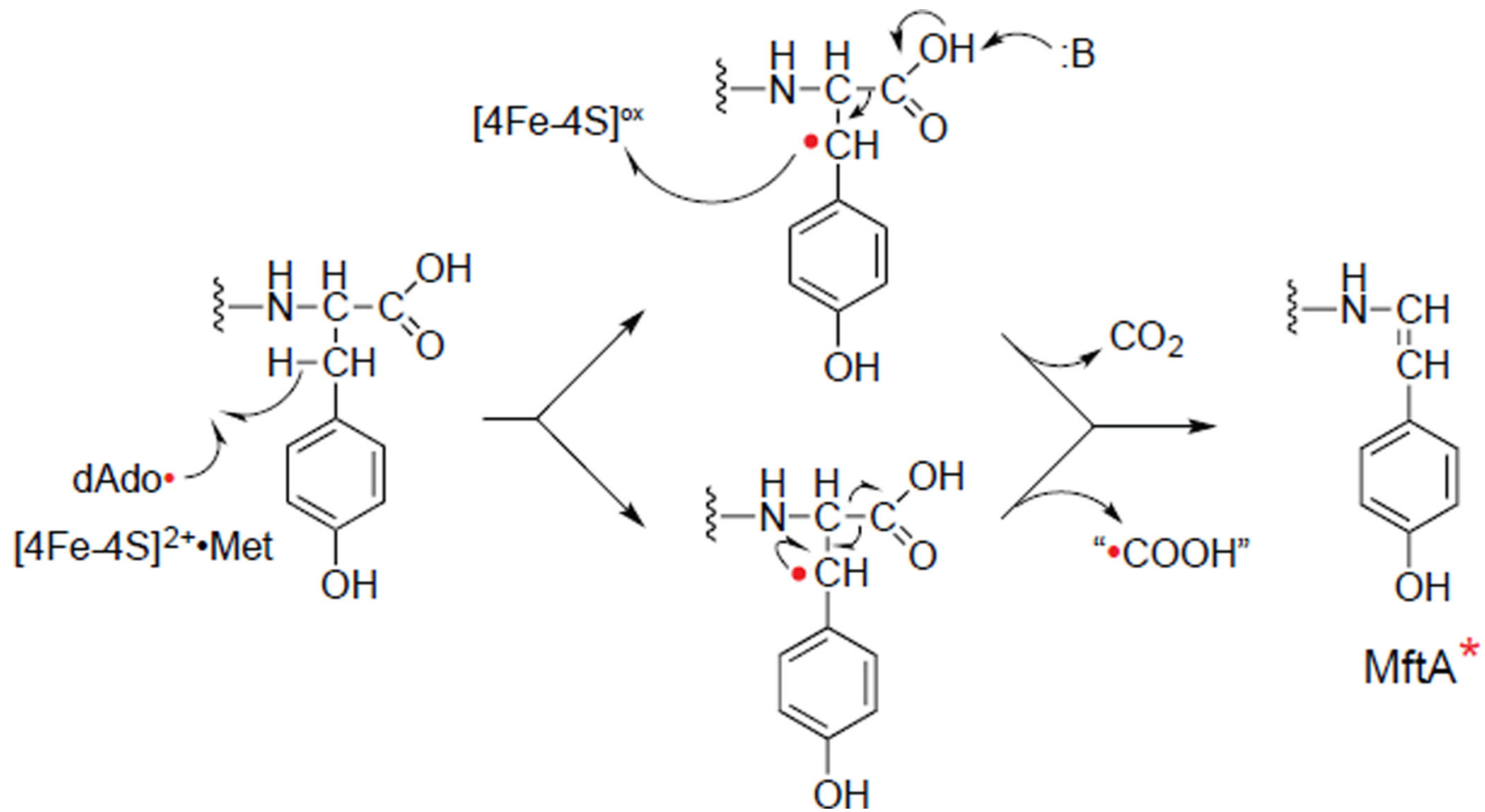

Figure 3.

Proposed catalytic mechanism for the oxidative decarboxylation of the C-terminus of MftA catalyzed by MftC. 


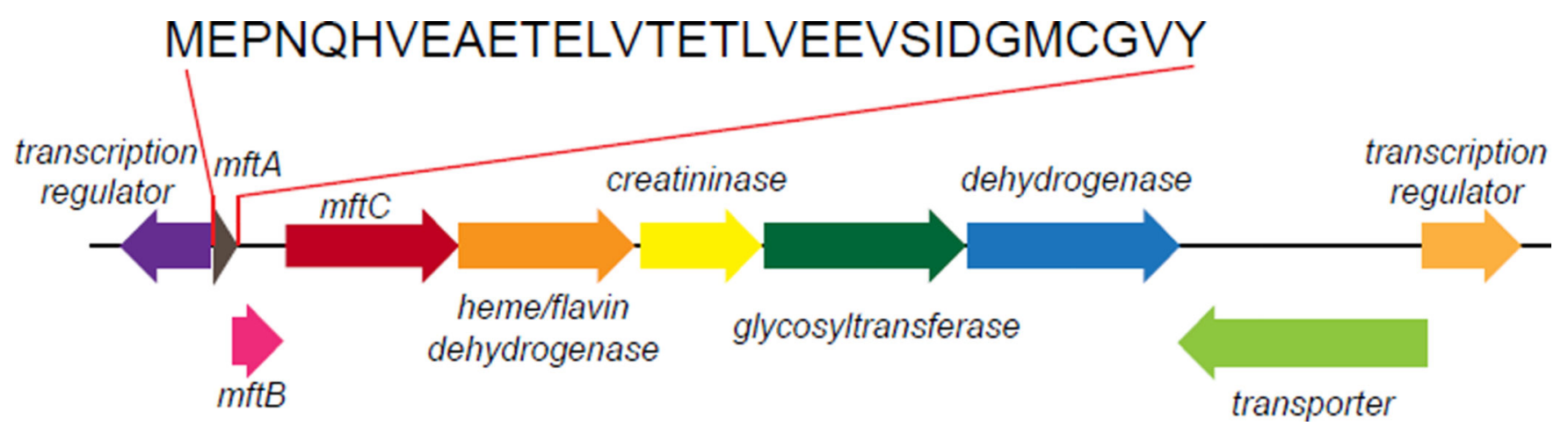

Scheme 1.

Mycofactocin gene cluster from Mycobacterium smegmatis ATCC 700084. The sequence of the peptide encoded by the $m f t A$ gene is shown. 